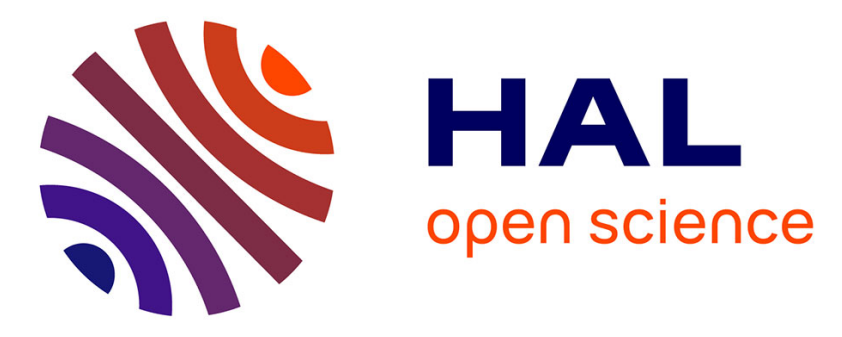

\title{
Causal Analysis Methodology of Multisensor Systems based on GNSS
}

\author{
Cyril Legrand, Julie Beugin, Blaise Conrard, Juliette Marais, Marion \\ Berbineau, El Miloudi El Koursi
}

\section{- To cite this version:}

Cyril Legrand, Julie Beugin, Blaise Conrard, Juliette Marais, Marion Berbineau, et al.. Causal Analysis Methodology of Multisensor Systems based on GNSS. Railways 2014, The Second International Conference on Railway Technology: Research, Development and Maintenance, Apr 2014, France. 21p. hal-00985634

\section{HAL Id: hal-00985634 https://hal.science/hal-00985634}

Submitted on 30 Apr 2014

HAL is a multi-disciplinary open access archive for the deposit and dissemination of scientific research documents, whether they are published or not. The documents may come from teaching and research institutions in France or abroad, or from public or private research centers.
L'archive ouverte pluridisciplinaire HAL, est destinée au dépôt et à la diffusion de documents scientifiques de niveau recherche, publiés ou non, émanant des établissements d'enseignement et de recherche français ou étrangers, des laboratoires publics ou privés. 


\title{
Causal Analysis Methodology of Multisensor Systems based on GNSS
}

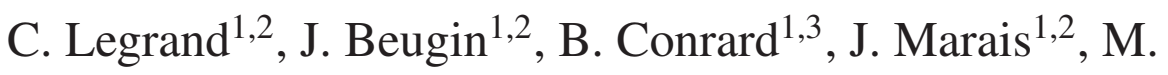 \\ Berbineau $^{1,2}$, and E.-M. El-Koursi ${ }^{1,2}$ \\ ${ }^{1}$ Lille Nord de France University, France
}

${ }^{2}$ The French Institute of Science and Technology for Transport, Development and Networks,

COmponents and SYStems Department (IFSTTAR-COSYS),

Villeneuve d'Ascq, France

${ }^{3}$ Laboratory of Automatics, Computer Engineering and Signal Processing (LAGIS), UMR

CNRS 8219,

Lille, France 


\begin{abstract}
For railway positioning solutions based on GNSS (Global Navigation Satellite Systems) like the GPS (Global Positioning System) or the future Galileo, a generic model is impossible to create in regards to the signal degradations in the atmosphere, the multipath effects caused by receiver near-environment, the multitude of environment configurations crossed by the train and the weak feedback of these technologies for estimated failure rates. The dependability assessment foundations of satellite-based positioning are laid in [1]. To compensate the weakness of GNSS, it must be hybridised with other sensors to determine a position sufficiently accurate for an use in safety applications [2]. A multitude of information sources is available about the train position. Only one position is possible. In consequence, a fusion step is necessary to combine all these sources of position. This raises some questions: Why the technology hybridisation is interesting to provide a accurate position? How the influences of sensor errors can affect the system output? Which sensors combination is the most efficient in regards to RAMS (Reliability, Availability, Maintainability and Safety) analyses required in railway safety standards? This paper proposes to focus on this last question with an analysis of different sensor architectures in order to understand how errors (propagation of failures) of one or several sensors can affect the entire positioning system. To answer to this question, a causal analysis is led based on the sensor behaviours.
\end{abstract}

Keywords: GNSS-based localisation system, Dependability, Multisensor system, Railway safety applications. 


\section{Introduction}

The first GNSS (Global Navigation Satellite Systems) - the GPS (Global Positioning System) - was designed by the DoD (Department of Defense) of United States for, first of all, aeronautics and military uses (missile guidance). Giving an unrivalled accuracy (in comparison with other positioning systems in this time) and a guaranteed independence to ground installations, a satellite system became a necessity to a military nation. That is why, Russia got equipped with the GLONASS (Global'naya Navigatsionnaya Sputnikovaya Sistema). In the mid of 1990s, the GPS became available to a civil use first in aeronautic domain and, nowadays, in ground transport (road and maritime transportation). In addition, face to the US monopoly, the European Union decided in 2002 to create their own GNSS, Galileo (full completion of the constellation expected by 2019). To more and more enhance the performances (especially in terms of accuracy and integrity (Ability of a system to timely alert the user of the unavailability of the service provided in the expected conditions)) of these technologies, overlay systems can be used in complementary of satellite systems like the EGNOS (European Geostationary Navigation Overlay Service) for an enhancement based on satellites or the DGPS (Differential GPS) based on ground installations.

These technologies show us their efficiency to provide an accurate position. However, the railway sector has been reluctant in the use of GPS in the past. Indeed, in a domain where the safety is more than required, a positioning service, which provide a fluctuated accuracy or can be interrupted (unavailability in a tunnel for example) is not acceptable. In addition, a step of certification is necessary based on a dependability assessment before putting into service a new positioning system based on GNSS.

Classically, the dependability is defined by the confidence on an expected service provided by a system. This confidence is addressed to the system designer but it is also useful for an user. The system will give a train position and an indicator, which indicates to him/her the confidence that can be placed into the position returned by the system. To determine the confidence, the knowledge of the error sensors is necessary. However, these data are lost after the fusion step. This is impossible to determine where the biases exactly come from in positioning system output and in which proportions.

According to the EN 50126 standard [3], the services of a railway system have to generate an acceptable level of safety and its implementation does not lead to an unacceptable features like an excessive failure rate.

In so doing i.e. to evaluate the dependability of a system, it exists two categories of methods: predictive and operational. In the predictive analyse, two approaches are possible : inductive and deductive. This paper will not establish a list of all existing methods but the focus is placed on a reasoning based on truth tables and decision trees, which permit to identify all the state of a system based on binaries 
behaviours [4]. These predictive methods require the perimeter definition of the qualitative/quantitative dependability analysis. This supposes that a physical hierarchy analysis has been done beforehand. In operational analyse, it is to characterise the function of a system and rank them. The interactions between sub-functions and the environment are also represented.

In a first part, a presentation of existing instrumentation system - sensors and techniques (proprioceptive and exteroceptive) - in railways will be made. These sensors architectures all of them are based on GNSS - are used in sereval projets (Rune [5], Grail-2 [6], Galoroi [7], etc). In the paper, the focus is placed on their fault modes and their impacts on the service returned by the system.

In a second part, an analysis will be made based on the combinatorial logic of sensors states. This step will be incremental. Indeed, different architectures will be considered with:

- an increased level of requirements in terms of RAMS attributes, accuracy or other indicators of performance

- a $1 \mathrm{D}$ and $3 \mathrm{D}$ context

- double or triple redundancies

In so doing, it proposes to begin with a simple theoretical case (three sensors : a GNSS receiver and two others) to a case closer to the reality. For each architectures and kind of faults leading to failure modes, expressions representing combination of logical states of sensors (nominal, acceptable or unacceptable) are computed. Furthermore, several detection techniques and combination strategies will be identified. Otherwise, the fusion step in multisensor positioning system will be supposed fault-free and correctly designed. The paper did not considered systematic errors.

This paper proposes to highlight behaviours of different multisensor systems with several architectures at the occurrence of one or several sensor errors. The main objective is to understand how a train positioning system is influenced by faults on its parts i.e. the sensors.

\section{Existing localisation systems for safety-related appli- cations}

\subsection{Railway positioning function}

The railway positioning function is realised by several sensors not just one. Nowadays, it does not exist a simple and an unique method to determine an accurate and sure position and guarantee it during all the time of a mission. That is why, a combination of absolute positioning, the vehicle position is determined by natural or artificial 
reference in environment (balises) and dead reckoning, the position is provided by on-board sensors is generally implemented. The proprioceptive sensors (used in dead reckoning) have a weak point: they suffer of cumulative errors. A bias occurs due to a slide and increase all along the time of a travel. However, the use of sensor, which provide an absolute position i.e. exteroceptive sensors can compensate this phenomena. The GNSS constitutes a absolute positioning solution and it is absolutely possible to integrate them in a railway domain. Nevertheless, are they reliable? Is their contribution significant and do they enhance existing positioning systems ?

\subsection{Evaluation of the localisation systems performances}

In addition to RAMS parameters, it exists an other parameter class specific to positioning system based on satellite technologies [1]: accuracy, continuity, integrity and availability. The accuracy is defined by the degree of conformity between the estimated position and position its real position. The continuity refers to the probability that the system continues to operate during the time of the mission/task considered. The integrity concept is already seen in the introduction. Finally, the availability concerned the time proportion during which the services provided by the system are available and conform to the requirements. As this class is exclusive to GNSS, an extension for a multisensor system is required and can lead to futures works.

The idea to use GNSS in railways is not new and it is the subject of several researches. In the next subsection, some project will be introduced.

\subsection{Projects about multisensor positioning system based on GNSS}

In this decade, several projects have been led to evaluate the contribution of satellite technologies in train positioning:

\subsubsection{APOLO (1998-2001) - Status: ended -}

The main objective APOLO (Advanced POsition LOcator) was to develop a train position locator (cf Figure 1) based GNSS-1 (i.e. GPS with EGNOS) and INS (Inertial Navigation System composed of odometer, gyroscope, accelerometer and Doppler inertial sensors commonly used in railways [8]) for proving its feasibility for railway safety applications.

Tests have been led with different experimental set-ups in order to validate the architecture's sensors in terms of accuracy and the error sources identification.

This architecture works with two sources of speed information in the same range of frequency. However, the odometer is the only proprioceptive sensor providing natively a position (even if, this information can be determined from speed but this operation 


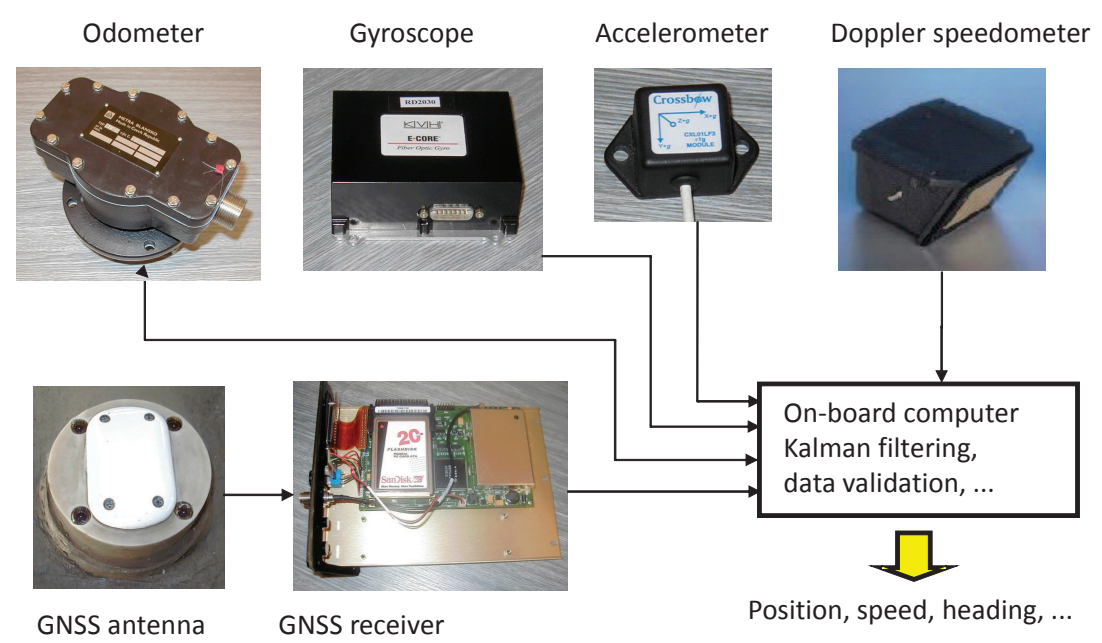

Figure 1: APOLO equipment

led to errors).

The conclusion of APOLO project was that this kind of system is not adapted for safety-critical applications on weak SIS (Signal In Space) availability lines. Indeed, after collecting data provided during tests, the gyroscope drift proves to be critical for this kind of applications. The error resulting from this phenomena leads to a degradation related to the accuracy of several ten of meters - beyond RAMS requirements give by [3] -.

The map-matching method (projection of position on precise map) has been given as a possibly reduction of this error.

\subsubsection{DemoOrt (2004-2007) - Status: ended -}

The DemOrt platform was built in order to evaluate the integration of different navigation systems especially innovative ones like GNSS on scenarios predefined (in open area and in a forest), applications where a safety responsibility is needed. Based on APOLO conclusions, GNSS can not be used alone for safety reasons. That is why, three parts compose this system (cf figure 2): 1) a GNSS (GPS) receiver, 2) RFID transponders and antennas, Doppler Radar and digital track map and 3) the software data processing with especially a fusion of information provided by the sensors except the GNSS part (qDemoOrt function). The fusion result is synchronised with GNSS receiver output.

In the dependability framework, the availability $(\boldsymbol{A})$ and safety are evaluated quantitatively throughout tests (110352 samples with GNSS output frequency of $2 \mathrm{~Hz}$ ) in 


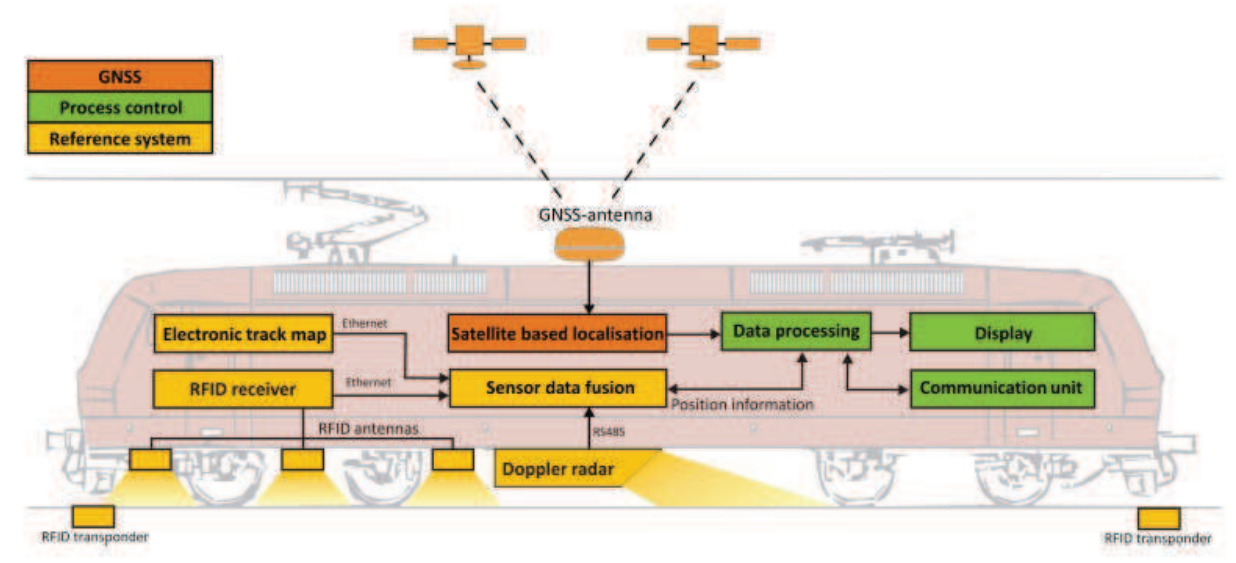

Figure 2: DemoOrt Structure [2]

the field by MTTF (Mean Time To Failure) measures and THR (Tolerable Hazard Rate) computation in each situation. For open area, $A=95.78 \%$ and $\boldsymbol{T H R}=$ $5.22 \cdot 10^{-7} /$ hour. For a forest area, $A=76.97 \%$ and $T H R=5.25$. $10^{-2} /$ hour.

The Doppler Radar constitutes the only on-board sensor. In consequence, its failure is critical. Indeed, in this case, the position would be determined by GNSS subsystem and the digital track map. In addition, this map does not represents an entire railways network (limited to the test tracks) and is determined a priori by GNSS services. The use of RFID technology [9] is interesting: it works at a range of frequencies bigger than railways balises.

As a proprioceptive sensor, Doppler Radar suffers of cumulative error and it represents a high drift. This is in this case that GNSS can be useful in readjusting the position.

Just after the first conclusions of the project, the ERTMS/ETCS compatibility had been discussed for futures works.

\subsubsection{RUNE (2001-2006) - Status: ended -}

The RUNE (Rail User Navigation Equipment) project [5] is funded by ESA in order to determine that satellite-based system especially using EGNOS can be used to localise a train and improvement in train driver's situational awareness. RUNE equipment regroups a GNSS receiver, an IMU ((Inertial Measurement Unit) and odometer. These equipment uses a Virtual Balises Map with balises ID and their 3D-position. Their goal is to enhance the self-capability of the train to determine its own position in order to reduce trackside equipment as much as possible. 


\subsubsection{GRAIL (2005-2007) - Status: ended - and GRAIL2 - Status: in progress (end: 12/2013) -}

The GRAIL [10] (and its extension GRAIL2 [6]) initiated by GSA in the Seventh Framework Programme, has as objectives to develop and validate a GNSS based ETCS (European Train Control System i.e.a component of ERTMS - (European Railway Traffic Management System)) application in High Speed Railway Lines. Among of the applications seen in the GRAIL project, GRAIL-2 focuses on Enhanced Odometry application with the use of GNSS subsystem as an additional sensor to compensate odometry problems in high speed runs (slip and slide phenomena). The project tried to validate this kind of system, which the composition is given in figure 3 (also used in GRAIL2 project) in a real environment with the constraints of urban area and the problems it generates.

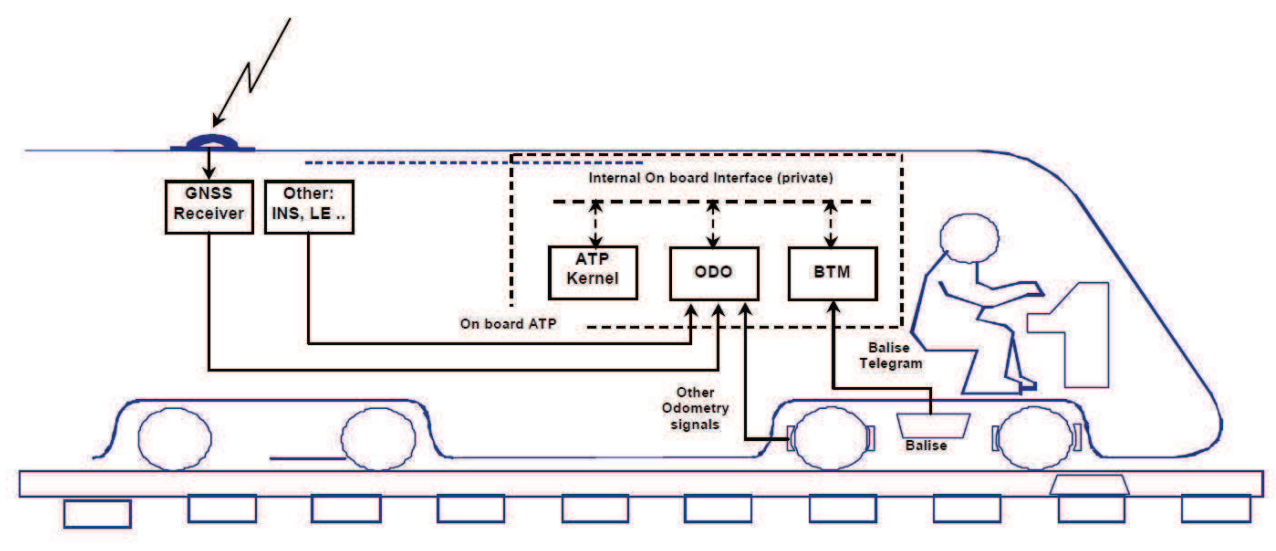

Figure 3: GRAIL prototype

The first conclusions are that GNSS data (position and speed) at low speed (15 $\mathrm{km} / \mathrm{h}$ ) in approaching of a station (Madrid station in the tests) are not enough accurate face to classicaly odometry data due to the weak GNSS reception in the test area. However, it has been shown that GNSS contribution became obvious on a long travel.

\subsubsection{InSat - Status: in progress (end: 2015) -}

The 3InSat (Train Integrated Safety Satellite System) project is funded by STS Ansaldo and ESA (European Space Agency). The main objectives are to develop a satellitebased platform compatible with ETCS in regards to the railways standards. Tests are ongoing in Sardania on a pilot line near Cagliari. The reference architecture (cf figure 4) is composed of GNSS (GPS and GLONASS) receiver, INS (Inertial Navigation System) and tachometer. These subsystems are called LDS (Location Determination System). From requirements in terms of LDS accuracy, THR (tolerable hazard rate) 
and availability, it had been possible to design a safe satellite-based train control system compatible with ERTMS/ETCS with the use of multi-constellations in a context of high integrity [11].

\section{InSat Reference Architecture}

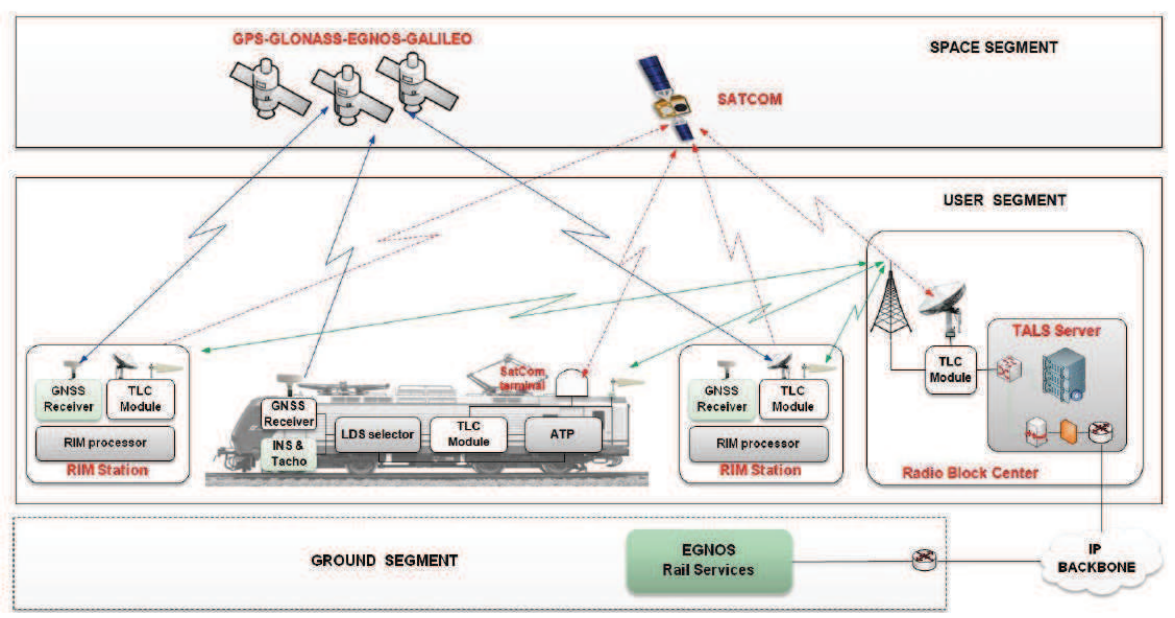

Figure 4: 3InSat Reference Architecture

\subsubsection{InteGRail (2005-2008) - Status: ended -}

In 2000, Kayser-Threde and Bombardier Transportation develop a prototype system InteGRail (for INTElligent inteGration of RAILway) providing a train position, velocity and heading by use of GNSS to safety-critical system. In ERTMS context, InteGRail platform (cf figure 5) is composed of inertial sensors (Odometer, Fibre optic gyroscope - Ars module in the figure - and accelerometer) combined with GPS receiver (enhanced with EGNOS system).

InteGRail system has been shown its efficiency in various operational conditions on low density line (rural lines). The most important integrity risk was identified as the multipath and shadowing influence on GNSS SIS.

\subsubsection{GaLoROI - Status: in progress (end: 2014) -}

In order to make durable the railway, the GaLoROI (Galileo Localization for Railway Operation Innovation) project propose the use of GNSS particularly the future Galileo in a context of low density traffic lines. The aim in this project is to design a certifiable satellite-based localisation (cf Figure 6). It is proposed to use, in addition with GNSS data, a Eddy Current Sensor (ECS), which is slide/slip-free - main disadvantages of proprioceptive sensors like odometer -. A safety layer is added to this architecture, 


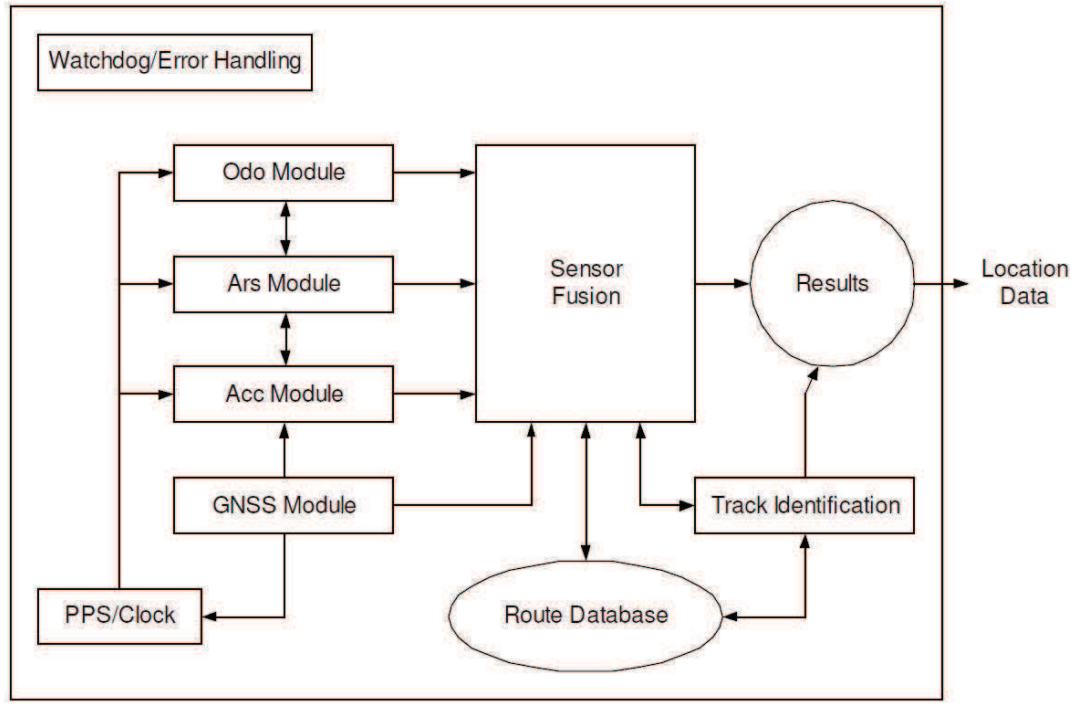

Figure 5: InteGRail platform [12]

which consists in the use of EGNOS Safety Of Life service providing timing and positioning signals (based on integrity data) for transport applications like railways.

This combination is performed by a fusion based on Extended Kalman Filter, a filter used a lot in navigation.

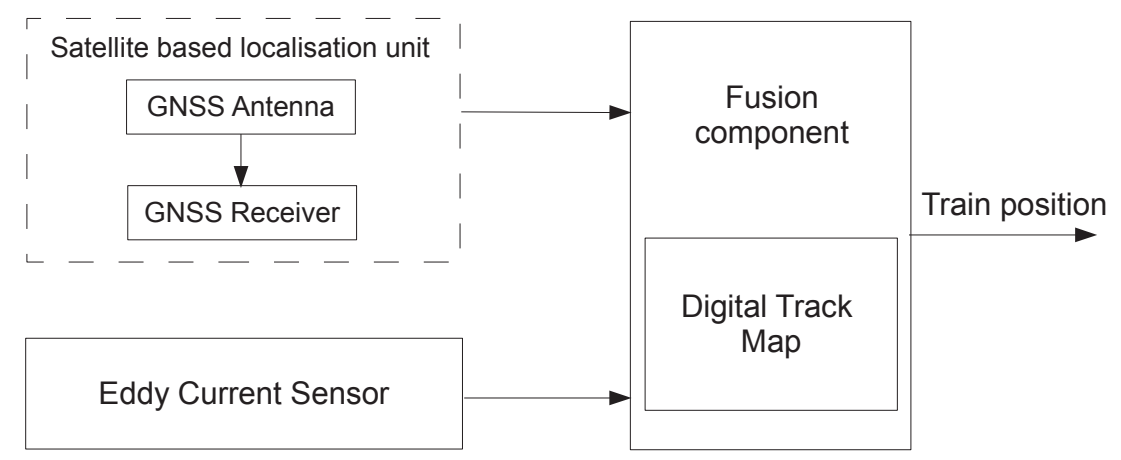

Figure 6: System concept used in GaloROI project [13]

All these projects conclude in the same way: GNSS is useful in railway localisation but their performance cannot meet the requirements in particular environment (forest, urban area). However, a hybridised solution with GNSS and existing on-board localisation system can be conceivable. 


\section{Causal analysis methodology for dependability qual- itative analysis of multisensor positioning systems}

\subsection{Methodology}

In this paper, the idea is to find any combinations of faults at the level of sensors, which lead to a failure of the entire multisensor system and determine which one is more efficient in terms of availability or safety. In so doing, a five-steps approach has been defined:

- The first step is to define the different states of sensor. Beyond the nominal and the fail state, possible faults on the sensors have to be identified: the faults related to an incorrect position provided by a sensor and the faults linked to the lack of information (no data or no response) about a sensor. In the case of an incorrect position, the output of a faulty sensor can be acceptable or unacceptable in regard to the accuracy requirement of the output. This step is common with all architecture.

- The definition of the detectability constitutes the second step. The state of a sensor can be detected or not. Following the case, it is a degradation related to the availability or the safety. For the detection of an intermittent fault (inspired by [14]), correlation between sensor measures are computed :

$$
\begin{array}{r}
0<r_{i 2} \text { and } r_{i 3} \text { and ... and } r_{i j}<0.5 \\
-0.5<r_{i 2} \text { and } r_{i 3} \text { and ... and } r_{i j}<0
\end{array}
$$

where, for the two sensors $i$ and $j, 0.5$ and -0.5 respectively represents a weak correlation in positive and negative direction between measures coming from sensor $i$ and $j$. It is computed by Eq 2 .

$$
r_{i j}=\frac{\sigma_{i j}}{\sigma_{i} \cdot \sigma_{j}}
$$

If a measure provided by a sensor $i$ is not correlated with the others, $i$ is considered faulty and it is removed from the fusion step.

For the detection and the treatment of the lack of information from a sensor has to be assessed. Three solutions can be found:

- the first solution is to remove the faulty sensor from the fusion step. For a n-sensor system and at the instant of the fault, the system is, actually, considered like a (n-1)sensor system. This solution is not recommended in the case of several faulty sensors or if the faulty sensor has a critical role (sensor devoted to readjust the other) 
- at the occurrence of the fault on a sensor, the last available data provided by the same sensor can be used for fusion. The fault effect is more distributed in time (cf Table 1). However, if this situation lasts a long time (the time until which for the position becomes unacceptable), the entire system has degraded performances (cf the "critical" combination in the fifth step).

- the available data of other sensor are used. This solution does not consist in the same thing than first one. Data are duplicated and replace incorrect one in fusion step.

In Table 1, these solutions are compared in regard to the accuracy on the final position after the fusion step. Data come from the first architecture in Section 3.2 .

\begin{tabular}{|l|c|}
\hline Solutions & Position error $(m)$ \\
\hline 1st solution: GPS is remove from the fusion process & 272,7085 \\
2nd solution: Last available data from the same sensor is used & 18,8046 \\
3th solution: If last available data from an other sensor is used & 5,4145 \\
\hline
\end{tabular}

Table 1: Influence of different fault treatment solutions on the accuracy

- The third step is the choice of the sensor architecture and the combination strategy. This is inspired by the different systems or prototypes seen in the projects presented before. All the architecture have to be composed of a GNSS receiver at least. Their use is decided in following step: Are GNSS data used at the same moment than the other sensor (involves that all the devices run at the same frequency) or when it is unavailable? About the combination itself, a fusion operator will be used (for example, a weighted average).

- In order to compare with the different simulations, the fourth step is a formalised example. Some assumptions are given: a set of measures provided by a given sensor follows a normal distribution centred on $\boldsymbol{\mu}$ value. $95 \%$ of the data $M_{i}$ are given in $\left[\mu_{i}-2 \cdot \sigma_{i} ; \mu_{i}+2 \cdot \sigma_{i}\right]=\left[\underline{M_{i}} ; \overline{M_{i}}\right]$. Whatever the kind of architecture, the multisensor system output is determined by the following interval (Eq. 3).

$$
[\underline{S} ; \bar{S}]=\left[f\left(\underline{C_{i}}\right) ; f\left(\overline{C_{i}}\right)\right]
$$

Let us consider $\boldsymbol{\beta}$, the accuracy specified as the position error at $95 \%$ confidence level and AlertLimit, the maximum allowable error in the measured position before an alarm is triggered (cf [3] and [15]. Knowing $\boldsymbol{\beta}<$ AlertLimit, the system output is considered as:

Acceptable ("N" state) if $[\underline{\boldsymbol{S}} ; \overline{\boldsymbol{S}}] \subset] \mathbf{0} ; \boldsymbol{\beta}[$ Degraded but Acceptable ("DA" state) if $[\underline{S} ; \bar{S}] \subset$ ] $\beta ;$ AlertLimit $[$ or 
$\boldsymbol{\beta} \geq \frac{\underline{S}+\bar{S}}{2}$

Unacceptable ("U” state) if $[\underline{S} ; \bar{S}] \subset]$ AlertLimit $+\infty[$ or AlertLimit $\geq$ $\frac{\underline{S}+\bar{S}}{2}$

An available position corresponding to a " $N$ " or " $D A$ " output state whatever this state is detected or not.

A safe position corresponding to a " $N$ " output state knowing that this state is detected.

If the conclusions of theoretical example and simulations converge, the sensor models used in these simulations are valid. A contrario, if a difference is noticed, the sensor models have to refined.

- In addition, we focus on the different possible logical combinations. Indeed, their persistence in time is important and change the state of the system output. For example, a " $(\boldsymbol{N}, \boldsymbol{D} \boldsymbol{A}, \boldsymbol{D} \boldsymbol{A})$ " combination can lead to a " $\boldsymbol{N}$ " output but, if this situation stays valid for several second (a time called thereafter "critical" time $\boldsymbol{t}_{c}$ ) this combination becomes " $\boldsymbol{D} \boldsymbol{A}$ ". This kind of combinations is called "critical" and represents output acceptability limit. Beyond this limit, the system is triggers a safe mode i.e. the train stops.

- For each sensor architecture, the steps three and four are realised. The last part of the analysis will be devoted to the comparison between the different systems and theoretical cases (cf fourth step) considered in regard to the efficiency of their fault tolerance technique i.e. the one, which impacts the least the accuracy.

In the next part, several architectures will be presented: sensors and fusion process.

\subsection{Sensor architectures considered}

This part begins with a simple theoretical case (cf Figure 7) already used in [16]. It

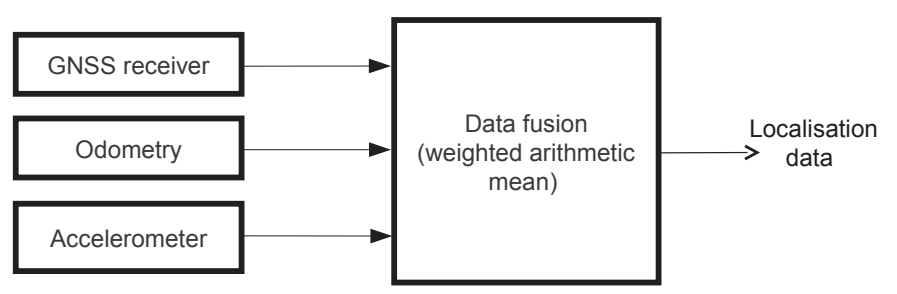

Figure 7: Accelerometer/Odometer/GPS receiver architecture

runs in an 1D-context (only the x-coordinate is considered). The fusion step consists of a weighted arithmetic mean computation. Each sensor is associated with a weight 
determined throughout the simulations by Eq. 4:

$$
\alpha_{i}=\frac{\sigma_{i}^{-2}}{\sum_{j=1}^{n} \sigma_{j}^{-2}}
$$

where, $\alpha_{i}$ is the weight of the sensor i, $\sigma_{i}$ is the standard deviation of the sensor i measures.

In multisensory fusion, this constitutes the easiest solution to use simultaneously all the position measures and does not need a long computing time.

This kind of multisensor system considered that each sensor provides a continuous position. Even if it is still correct for proprioceptives (odometer and accelerometer in this case), GPS receiver provides discrete data. However, extrapolation is possible when there is no data from GPS receiver.

An other architecture can be imagined with the same sensors (cf Figure 8). Here, the proprioceptive sensors are readjusted by GPS data. In the case of GPS SIS unavailability, only odometry and accelerometer measures are treated because the readjustment is not available. This solution is classically used in navigation [17]. Data in Figure 8 are considered to be in the same format (conversion or other transformation are implicit - operation is located inside sensor boxes in the figure).

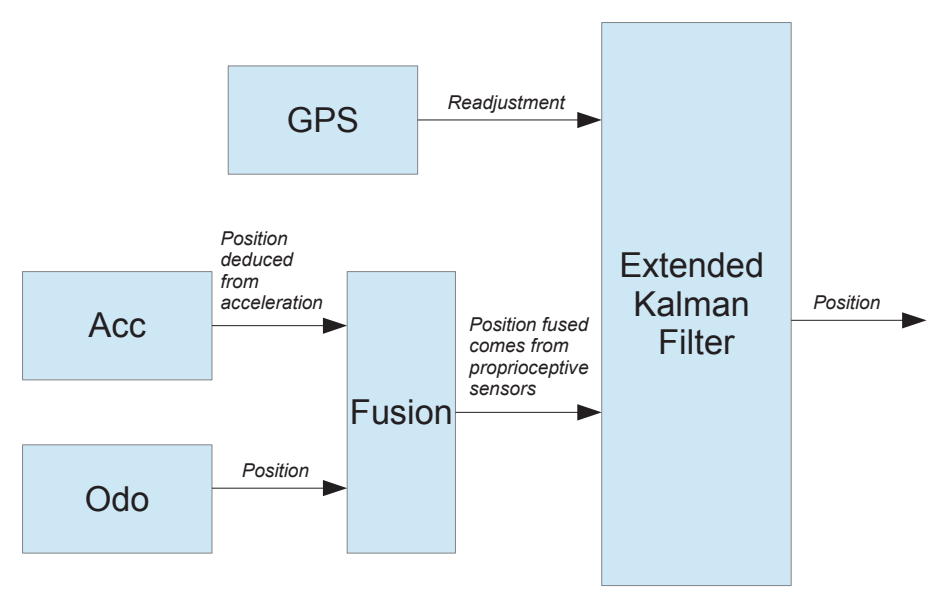

Figure 8: Accelerometer/Odometer/GPS receiver enhanced architecture with Extended Kalman Filter

For the transition between 1D-context and 3D-context, the accelerometer is replaced by an IMU. Classically, it is just three accelerometers - one for each coordinate in Cartesian space -. Therefore, to have a correct position, all its coordinates have to be correct. 
To enhance the reliability of a system, the redundancy is often used. In a railway localisation, the redundancy can be applied at different levels: the multi-constellation (cf 3InSat project) or the multiplication of on-board sensors. It is very current to find an odometer on several wheels or axles to mitigate the slip/slide effect. However, some redundancies can not be realistic especially in terms of costs. Some sensor like INS are very expensive. In consequence, for a fourth architecture, only a double redundancy and is considered.

\subsection{Causal analysis of each systems}

Each path of the tree (Figure 9) represents a logical combination of sensor states. Green, Orange, Red paths represent respectively logical combinations leading to a "N", "DA" and "U" output state.

These results correspond to the theoretical case (cf Section 3.1 in fourth step) with high requirements in regards to level of accuracy. This means that the $\boldsymbol{\beta}$ and AlertLimit have been fixed respectively to 1 and 2,5 meters (requirements for train control on high density line [2]).

Among to the " $\boldsymbol{D} \boldsymbol{A}$ " combination (20 in total), 15 combinations are considered as "critical". Indeed, in these cases, if the middle of $[\underline{S} ; \bar{S}]$ i.e. $\frac{\underline{S}+\bar{S}}{2}$ is upper to AlertLimit value, this last becomes unacceptable.

For the sake of size of the paper, this decision tree is not shown for each architecture but for the formal example presented in Section 3.1 step four.

Insofar as possible, a mathematical model is programming to simulate the behaviour of each sensor. For example, accelerometer model is given in [18] and use in the simulation. The other sensors model (for odometer and GPS receiver) are explained in [16].

In the case of sensor hardware failures, the distribution for the time $t$ of a failure occurrence is approximated by an exponential law.

\subsubsection{Architecture 1: Accelerometer + Odometer + GPS receiver}

The architecture considered here is presented in Figure 7. Eight combinations (cf Table 2) are identified as "critical" i.e. their persistence in time (after $\boldsymbol{t}_{c}$ second(s)) can change the output system from " $D A$ " to " $U$ " state.

In addition to $\boldsymbol{U}$ ones, these combinations are to keep under surveillance. They represent the limit of position acceptability. As an example, for this architecture, if 


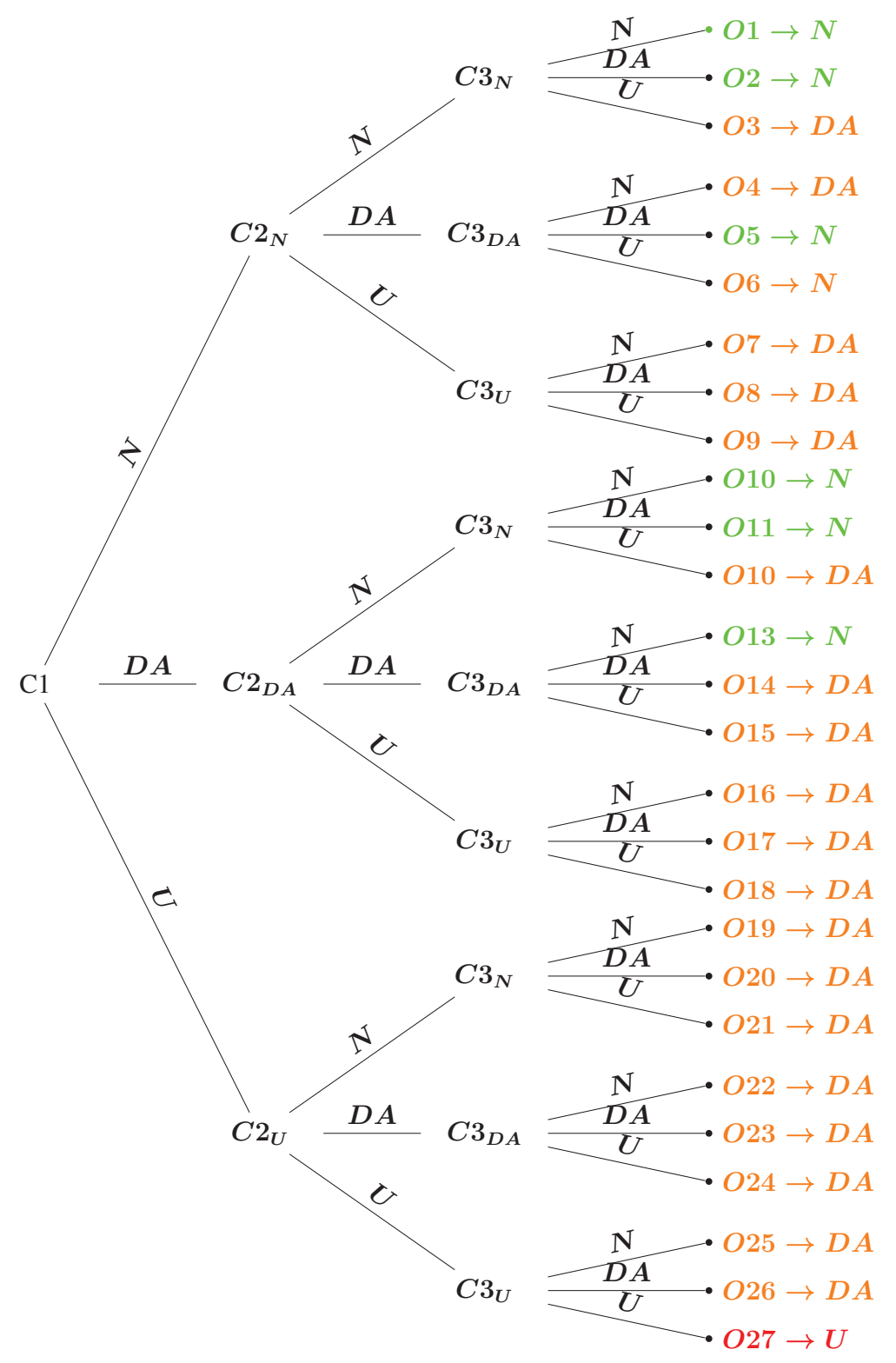

Figure 9: Decision tree for a 3-sensors architecture

$(\boldsymbol{U}, \boldsymbol{U}, \boldsymbol{N})$ combination of sensors state are still the same after $\boldsymbol{t}_{c}=2$ seconds, the output state changes from " $D A$ " to " $U$ ".

The $(\boldsymbol{N}, \boldsymbol{N}, \boldsymbol{N}),(\boldsymbol{N}, \boldsymbol{N}, \boldsymbol{D} \boldsymbol{A})$ and $(\boldsymbol{N}, \boldsymbol{D} \boldsymbol{A}, \boldsymbol{N})$ combinations, lead well to a $\boldsymbol{N}$. So, they respect the safety theorem (no "U" in the combinations). 7 combinations lead to a "U" output whatever happens. 


\begin{tabular}{|c|}
\hline Combination format : \\
(state(Odo), state $($ Acc $)$, state $(\mathrm{GPS}))^{1}$ \\
\hline$(\boldsymbol{D} \boldsymbol{A}, \boldsymbol{D} \boldsymbol{A}, \boldsymbol{D} \boldsymbol{A})$ \\
$(\boldsymbol{U}, \boldsymbol{N}, \boldsymbol{D} \boldsymbol{A})$ \\
$(\boldsymbol{U}, \boldsymbol{D} \boldsymbol{A}, \boldsymbol{N})$ \\
$(\boldsymbol{D} \boldsymbol{A}, \boldsymbol{U}, \boldsymbol{N})$ \\
$(\boldsymbol{D} \boldsymbol{A}, \boldsymbol{N}, \boldsymbol{U})$ \\
$(\boldsymbol{N}, \boldsymbol{D} \boldsymbol{A}, \boldsymbol{U})$ \\
$(\boldsymbol{N}, \boldsymbol{U}, \boldsymbol{D} \boldsymbol{A})$ \\
$(\boldsymbol{U}, \boldsymbol{U}, \boldsymbol{N})$ \\
\hline
\end{tabular}

Table 2: "Critical" combinations of architecture 1

\subsubsection{Architecture 1 bis: Accelerometer + Odometer + GPS receiver + EKF}

In this architecture, the GPS receiver is used for the readjustment accelerometer and odometer data. The last sensors follow the same models than the first multisensor system.

The principle of the Extended Kalman Filter (EKF) is not explained in this paper. However, to understand the GPS contribution, the Kalman filter has two main steps: prediction and update. GPS receiver data are used in this last step. The EKF is considered as fault-free.

The "critical" combinations are the ones where the GPS receiver state is to " $U$ ". In consequence, it concerns $(\phi, \phi, U)$ with $\phi=\{\boldsymbol{N}, \boldsymbol{D} \boldsymbol{A}, \boldsymbol{U}\}$. Indeed, if the readjustment is strongly degraded, the step of update will be biased. the $t_{c}$ time is $\mathbf{1}$ to $\mathbf{3}$ seconds depending on the importance of the degradation.

Finally, this architecture is very dependent on the performances of GPS receiver. In real case, degradations related to GPS SIS are correlated to the environment configuration like urban or forest. These situation correspond to $(\phi, \phi, U)$ combinations.

\subsubsection{Architecture 1 ter: Accelerometer + Odometer x2 + GPS receiver}

Here, the odometer is duplicated to implement an active redundancy. This choice is explained in Section 3.2. The two odometers have the same features (resolution, noises, etc... (cf [16])).

In this architecture (a 4 -sensor system), there are, in consequence, $3^{4}$ so 81 possible logical combinations of sensor states. They are not all occurred during the simulation. Table 3 shows the combinations that occurred during a simulation of the architecture (100 seconds). 


\begin{tabular}{|c|}
\hline Combination format : \\
(state(Odo),state(Odo2), state(Acc),state(GPS)) \\
\hline$(\boldsymbol{U}, \boldsymbol{U}, \boldsymbol{N}, \boldsymbol{D} \boldsymbol{A})$ \\
$(\boldsymbol{U}, \boldsymbol{U}, \boldsymbol{D} \boldsymbol{A}, \boldsymbol{D} \boldsymbol{A})$ \\
$(\boldsymbol{U}, \boldsymbol{U}, \boldsymbol{U}, \boldsymbol{N})$ \\
$(\boldsymbol{D} \boldsymbol{A}, \boldsymbol{D} \boldsymbol{A}, \boldsymbol{D} \boldsymbol{A}, \boldsymbol{D} \boldsymbol{A})$ \\
$(\boldsymbol{D} \boldsymbol{A}, \boldsymbol{D} \boldsymbol{A}, \boldsymbol{U}, \boldsymbol{N})$ \\
$(\boldsymbol{D} \boldsymbol{A}, \boldsymbol{D} \boldsymbol{A}, \boldsymbol{N}, \boldsymbol{U})$ \\
$(\boldsymbol{N}, \boldsymbol{N}, \boldsymbol{D} \boldsymbol{A}, \boldsymbol{U})$ \\
$(\boldsymbol{N}, \boldsymbol{N}, \boldsymbol{U}, \boldsymbol{D A})$ \\
\hline
\end{tabular}

Table 3: "Critical" combinations of architecture 1 ter

First conclusions, the "critical" combinations where the odometer provides an unacceptable position are not critical if the second odometer give a nominal value.

\subsubsection{Architecture 2: IMU + Odometer + GPS receiver}

The IMU is considered as 3 accelerometers, one on each axis. The features of IMU depend on the accelerometers seen in previous architectures. In addition, each component of the position vector ( $\mathrm{x}, \mathrm{y}$ and $\mathrm{z}$ in Cartesian coordinate system) have to be correct to provide a $3 \mathrm{D}$ correct position. This consideration is taking into account in IMU model for the simulation via its parameters.

\begin{tabular}{|c|}
\hline Combination format : \\
(state(Odo), state(IMU), state(GPS) $)$ \\
\hline$(\boldsymbol{U}, \boldsymbol{U}, \boldsymbol{N})$ \\
$(\boldsymbol{U}, \boldsymbol{U}, \boldsymbol{D} \boldsymbol{A})$ \\
$(\boldsymbol{U}, \boldsymbol{U}, \boldsymbol{U})$ \\
$(\boldsymbol{D} \boldsymbol{A}, \boldsymbol{D} \boldsymbol{A}, \boldsymbol{D} \boldsymbol{A})$ \\
$(\boldsymbol{D} \boldsymbol{A}, \boldsymbol{D} \boldsymbol{A}, \boldsymbol{U})$ \\
$(\boldsymbol{D} \boldsymbol{A}, \boldsymbol{D} \boldsymbol{A}, \boldsymbol{N})$ \\
$(\boldsymbol{N}, \boldsymbol{N}, \boldsymbol{D} \boldsymbol{A})$ \\
$(\boldsymbol{N}, \boldsymbol{N}, \boldsymbol{U})$ \\
\hline
\end{tabular}

Table 4: "Critical" combinations of architecture 2

The first observation is that the $(\boldsymbol{U}, \boldsymbol{U}, \boldsymbol{U})$ combination does not immediately lead to a $\boldsymbol{U}$ output. However, in this case, $\boldsymbol{t}_{c}$ is very low i.e. 1 second. A precaution must be taken: here, the time step is 1 second. For the worst "critical" combinations i.e. the combinations with a $t_{c}$ time very short $\left(t_{c} \leq 1\right)$, a time step must be more adequate i.e. less than the shortest $\boldsymbol{t}_{c}$ time. 


\section{Conclusions and prospects}

In this paper, a causal analysis of several multisensor systems based on GNSS has been attempted. These lasts are inspired by the different platforms already used in projects presented at the beginnings of the paper.

"Critical" combinations have been identified for several sensor architecture, combinations, which if they persist after a time (called $\boldsymbol{t}_{c}$ ) became unacceptable.

The time determined for a "critical" combinations can be seen as a maximum Time To Alarm (quantity used to evaluate the integrity, attribute only valid for the GNSS performances). This constitutes a possible prospect for an integrity concept extended to an entire multisensor system in order to assess its performances.

All the times are not measured yet. The immediate pursuance of this paper is to determined the times $\boldsymbol{t}_{\boldsymbol{c}}$ for each "critical" combinations.

In future works, an analyse of multisensor system behaviour will be made in existing environment with data (true measures of different sensors seen in this paper).

A potential perspective is to use importance [4] and sensitivity measures [16] in order to quantify the sensor error influences.

\section{Acknowledgments}

The authors would like to thank the Technological Research Institute Railenium and the IFSTTAR for their support in this work and the International Campus on Safety and Intermodality in Transportation (CISIT) and the European Commission via the European Regional Development Fund (ERDF) for making possible this work.

\section{References}

[1] J. Beugin, J. Marais, and J.-P. Lozac'h, "A dependability analysis for integrating a satellite positioning system in a rail freight application," in European Navigation Conference, 2008.

[2] D. Lu, F. Grasso Toro, and E. Schnieder, "Rams evaluation of gnss for railway localisation," in Intelligent Rail Transportation (ICIRT), 2013 IEEE International Conference on, 2013, pp. 209-214.

[3] EN 50126 Railway Applications: The specification and demonstration of Reliability, Availability, Maintenability and Safety (RAMS)., European Committee for Electrotechnical Standardization (CENELEC) Std., 2000. 
[4] M. Rausand and A. Høyland, System Reliability Theory: Models, Statistical Methods and Applications, Second Edition. Wiley-Interscience, 2003.

[5] "Railway User Navigation Equipment (RUNE) project," 2006. [Online]. Available: http://www.esa.int/Our_Activities/Navigation/Railway_User_Navigation_ Equipment_RUNE2

[6] "Grail-2 project," 2012. [Online]. Available: http://grail2.ineco.es/Grail2/html/ main.jsp

[7] “GaLoROI project.” [Online]. Available: http://www.galoroi.eu/

[8] J. Liu, T. Tang, B. Gai, J. Wang, and D. Chen, "Integrity assurance of gnss-based train integrated positioning system," Science China, vol. 54, p. 14, 2011.

[9] S. A. Weis, "Rfid (radio frequency identification): Principles and applications."

[10] GSA, "GNSS introduction in the RAIL sector: GNSS subsystem requirement specification for enhanced ETCS applications," Project funded by the European GNSS Supervisory Authority, 6th framework program, Tech. Rep., 2007.

[11] F. Rispoli, A. Filip, M. Castorina, G. Di Mambro, A. Neri, and F. Senesi, "Recent progress in application of gnss and advanced communications for railway signaling," in Radioelektronika (RADIOELEKTRONIKA), 2013 23rd International Conference, 2013, pp. 13-22.

[12] S. Bedrich and X. Gu, "GNSS-Based Sensor Fusion for Safety-Critical Applications in Rail Traffic," Galileo and EGNOS Information Catalogue, p. 8, 2004.

[13] T. Nguyen, J. Beugin, and J. Marais, "Dependability evaluation of a gnss \& ecs based localisation unit for railway vehicles," in 13th International Conference on ITS Telecommunication (ITST 2013), vol. Proceedings of 13th Internation Conference on ITS Telecommunication (ITST 2013), 2013.

[14] P. Caspi and R. Salem, Threshold and Bounded-Delay Voting in Critical Control Systems, 2000, ch. Formal Techniques in Real-Time and Fault-Tolerant Systems, pp. 70-81.

[15] G. Barbu, P. Frsig, M. Schrder, C. Edwards, K. Walter, A. Filip, A. Sage, and S. Forsyth, "GNSS RAIL USER FORUM REQUIREMENTS OF RAIL APPLICATIONS,” 2000. [Online]. Available: http://citeseerx.ist.psu.edu/ viewdoc/download?doi=10.1.1.113.148\&rep=rep1\&type=pdf

[16] C. Legrand, J. Beugin, B. Conrard, J. Marais, M. Berbineau, and E.-M. ElKoursi, "Sensitivity assessment to analyse dependability of a multisensor localisation system based on gnss," in 13th International Conference on ITS Telecommunication (ITST 2013), 2013. 
[17] D. Mayhew McNail, "Multi-rate sensor fusion for gps navigation using kalman filtering," Ph.D. dissertation, Virginia Polytechnic Institute, 1999.

[18] D. Kubrak, "Hybridisation of a GPS Receiver with Low-Cost Sensors for Personal Positioning in Urban Environment," Ph.D. dissertation, Telecom Paris, 2007. [Online]. Available: http://citeseerx.ist.psu.edu/viewdoc/download?doi= 10.1.1.113.148\&rep=rep1\&type=pdf 